\title{
Stereotactic radiosurgery and stereotactic body radiation therapy cost-effectiveness results
}

\author{
Akash Bijlani ${ }^{1}$, Giovanni Aguzzi ${ }^{2}$, David W. Schaal ${ }^{1}$ and Pantaleo Romanelli ${ }^{2}$ * \\ ${ }^{1}$ Accuray Incorporated, Sunnyvale, CA, USA \\ ${ }^{2}$ CyberKnife Center, Centro Diagnostico Italiano, Milan, Italy
}

\section{Edited by:}

Brian T. Collins, Georgetown Hospital, USA

\section{Reviewed by:}

Anatoly Dritschilo, Georgetown University School of Medicine, USA

Sean Collins, Georgetown University Hospital, USA

\section{${ }^{*}$ Correspondence:}

Pantaleo Romanelli, Brain

Radiosurgery, CyberKnife Center,

Centro Diagnostico Italiano, Via Saint

Bon 20, 20147, Milan, Italy;

European Synchrotron Radiation

Facility, Grenoble, France.

e-mail: radiosurgery2000@yahoo.com
Objective: To describe and synthesize the current stereotactic radiosurgery (SRS) and stereotactic body radiation therapy (SBRT) cost-effectiveness research to date across several common SRS and SBRT applications.

Methods: This review was limited to comparative economic evaluations of SRS, SBRT and alternative treatments (e.g., other radiotherapy techniques or surgery). Based on PubMed searches using the terms, "stereotactic," "SRS," "stereotactic radiotherapy," "stereotactic body radiotherapy," "SBRT," "stereotactic ablative radiotherapy," "economic evaluation," "quality adjusted life year (QALY)," "cost," "cost-effectiveness," "cost-utility," and "cost analysis," published studies of cost-effectiveness and health economics were obtained. Included were articles in peer-reviewed journals that presented a comparison of costs between treatment alternatives from January 1997 to November 2012. Papers were excluded if they did not present cost calculations, therapeutic cost comparisons, or health economic endpoints.

Results: Clinical outcomes and costs of SRS and SBRT were compared to other therapies for treatment of cancer in the brain, spine, lung, prostate, and pancreas. Treatment outcomes for SRS and SBRT are usually superior or comparable, and cost-effective, relative to alternative techniques.

Conclusion: Based on the review of current SRS and SBRT clinical and health economic literature, from a patient perspective, SRS and SBRT provide patients a clinically effective treatment option, while from the payer and provider perspective, SRS and SBRT demonstrate cost savings.

Keywords: cost-effectiveness, health economics, stereotactic body radiotherapy, stereotactic body radiation therapy, stereotactic radiosurgery, cancer

\section{INTRODUCTION}

For over 40 years clinicians have treated intracraniallesions with stereotactic radiosurgery (SRS). In the beginning, this noninvasive, highly innovative technique was received with great skepticism by the leaders of academic neurosurgery and radiation oncology. After 20 years of successful use in Sweden, the first Gamma Knife treatment was performed in North America in 1987 at the University of Pittsburgh. In 1989, the Gamma Knife was first used to treat the most common brain pathology, brain metastases (Lindquist, 1989). Since that time, radiosurgery has been shown to have excellent clinical outcomes and to be a costeffective treatment option for patients with brain metastases (Lee et al., 2009).

Starting in 1995, Blomgren et al. (1995) and Hamilton et al. (1995) provided the first results of radiosurgical techniques outside of brain and spine in a procedure that has come to be called stereotactic body radiation therapy (SBRT). SBRT is the imageguided delivery of high dose radiation in an extremely hypofractionated treatment (typically up to five fractions). Delivering such high doses per fraction requires high conformality and steep dose fall-off to avoid irradiating organs at risk; this necessitates imageguidance for patient setup and, preferably, throughout treatment to adjust for changes in tumor/target position, thus minimizing treatment-related toxicity. Advances in image-guidance have allowed clinicians to safely deliver both SRS and SBRT, and as the growing body of literature has supported the safety and efficacy of these procedures, their utilization has steadily increased.

Before the advent of frameless techniques, Gamma Knife was primarily used to deliver SRS. Although it is an effective radiosurgery device, it is limited to treating only intracranial and upper spinal lesions due to the necessity of a rigid head frame. The frame also effectively limits the Gamma Knife to single-session (or fraction) treatment. For intracranial lesions, fractionated SRS may provide additional normal tissue protection when treating tumors near functional regions such as the optic chiasm or inner ear (Chang et al., 2005; Adler et al., 2006). The CyberKnife was built on the principles of Gamma Knife radiosurgery, delivering both isocentric treatments (like Gamma Knife) and non-isocentric treatments, but does not require a rigid head frame. Instead frequent image-guidance is used to locate the target (in some cases 
based on the position of implanted fiducial markers) and adjust the beam aim should any changes in target position be detected. The CyberKnife is also able to track and automatically correct for respiratory motion of targets in lung, liver, pancreas, etc. Although the CyberKnife was designed specifically for SRS/SBRT, SBRT, and SRS can also be delivered by gantry-based radiotherapy systems (e.g., Varian Truebeam, BrainLab's Novalis TX, or BrainLab/Mitsubishi Vero). These systems also employ image-guidance for patient setup and, in some cases, occasional intra-fraction verification of target position, in addition to some combination of patient and target restraint using body frames and abdominal compression devices, breathing control, or respiratory gating to manage respiratory motion, and implanted fiducials or electromagnetic beacons.

As SRS and SBRT have grown, so has the clinical literature describing its application in treating a variety of tumors and lesions throughout the body including those in the brain, head/neck, spine, lung, liver, prostate, and pancreas. Despite the growing body of clinical SRS and SBRT literature, there is limited research into the cost-effectiveness and health economic outcomes of these procedures. Our long-term goal is to develop valid health economic research on SBRT and SRS; the current paper aims to describe and synthesize the SRS and SBRT cost-effectiveness research to date for several common SRS/SBRT indications.

\section{METHODS}

\section{SEARCH STRATEGY}

Based on a PubMed search using the terms, "stereotactic," "SRS," "stereotactic radiotherapy," "stereotactic body radiotherapy," "SBRT," "stereotactic ablative radiotherapy," "economic evaluation," "quality adjusted life year (QALY)," "cost," "costeffectiveness," "cost-utility," and "cost analysis," published studies of cost-effectiveness and health economics were obtained. Inclusion criteria were limited to articles in published peer-reviewed journals and needed to include a comparison of costs between alternatives from January 1997 to November 2012.

\section{INCLUSION/EXCLUSION CRITERIA}

This review includes only comparative studies of SRS, SBRT, and alternative treatments in economic evaluations. Inclusion criteria were limited to articles in published peer-reviewed journals and needed to include a comparison of costs between alternatives from January 1997 to November 2012. Exclusion criteria included the absence of cost calculations, therapeutic cost comparisons, and health economic endpoints. Title, abstracts and full-text articles of all identified studies were reviewed independently by two co-authors.

\section{BRAIN}

There are several published cost-effectiveness studies that focus on the clinical efficacy and cost-effectiveness of SRS compared to surgery (Table 1). One of the main reasons for this is that patients are treated with SRS on an outpatient basis compared with surgery, which requires utilization of inpatient hospital resources. Vuong et al. (2013) found that the average cost in Germany per patient for surgical resection was $€ 11,647$ compared to $€ 9,964$ for SRS. In addition, the survival time for surgical resection was 13.0 months while the survival time for SRS was 18.4 months. Also in Germany,
Wellis et al. (2003) calculated the treatment costs of SRS and microsurgery for the treatment of meningiomas, acoustic neuromas, metastases, and arteriovenous malformations. For microsurgery, the average hospitalization time was $15.4 \pm 8.6$ days with $1.2 \pm 2.8$ of those days spent in the intensive care unit (ICU). The total average costs of microsurgery per patient including ancillary therapy and unplanned readmissions was $€ 15,252$, while the total average cost of SRS per patient was $€ 7,920$. Along the same lines, in Netherlands, van Roijen et al. (1997) analyzed costs and effects of treating acoustic neuroma patients with either microsurgery or radiosurgery. Direct costs for microsurgery were Dfl. 20,072 and Dfl. 14,272 for radiosurgery, while indirect costs were Dfl. 16,400 for microsurgery and Dfl. 1,020 for radiosurgery. In addition, the general health rating was better for radiosurgery than for microsurgery. Banerjee et al. (2008) also compared the costs of microsurgery to radiosurgery for the treatment of vestibular schwannoma. For microsurgery patients who were followed up for at least 36 months, mean surgical costs were $\$ 23,788$, while for radiosurgery patients, the mean surgical costs were $\$ 16,143$. For microsurgery patients, the mean follow-up costs per month started at over $\$ 1,000$ per month and decreased steadily to less than $\$ 70$ per month by the tenth month of follow-up. The mean followup costs for patients in the radiosurgery group were less than $\$ 10$ per month for the first few months and thereafter increased to as much as $\$ 200$ per month. In addition, the microsurgery patients suffered a significant decline from pre-operative levels in several components of the health status questionnaire (HSQ) at 3 months, 1 year, and most-recent follow-up; however, the radiosurgery group showed no decline in HSQ across all follow-up time frames.

Manning et al. (2000) compared the treatment cost of linacbased hypofractionated stereotactic radiotherapy (HSRT) and SRS for the treatment of brain metastases. The median absolute cost of SRS was $\$ 4,119$ higher than HSRT. In Taiwan, Cho et al. (2006) compared the direct and indirect costs from both hospital and societal perspectives for SRS and open surgery for the treatment of benign cranial base tumors. For open surgery, the mean length of stay was 18.2 \pm 30.4 days including $5.0 \pm 14.7$ days of ICU stay and $13.0 \pm 15.2$ days of ward stay. The mean hospital stay for SRS was $2.2 \pm 0.9$ days with no need of ICU stay. The mean loss of workdays for open surgery was $160 \pm 158$ and $8.0 \pm 9.0$ days for SRS. The direct cost for SRS was higher than that for open surgery $(\$ 9,677 \pm \$ 6,700$ vs. $\$ 5,837 \pm 6,587)$. Open surgery had a higher complication rate $(31.2 \%)$ compared to SRS (3.8\%). Open surgery had a mortality rate of $5.3 \%$ while there was no mortality for SRS. The socioeconomic costs were significantly higher for open surgery compared to SRS $(\$ 34,453 \pm 97,277$ vs. $\$ 10,044 \pm 7,481)$. Finally, the cost per QALY was significantly lower with SRS compared to open surgery ( $\$ 3,762 / \mathrm{QALY}$ vs. $\$ 8,996 / \mathrm{QALY})$. Along the same lines, Tarricone et al. (2008) compared the full treatment costs of SRS vs. microvascular decompression (MVD) for trigeminal neuralgia. The MVD full treatment costs were $€ 6,641$ per patient while the full SRS treatment costs were $€ 4,388$ per patient. The difference was attributed to the cost of the surgical procedure and the cost of inpatient hospitalization for MVD, which was, on average, 10 days (no hospitalization is required for SRS). Lal et al. (2012) utilized a decision analysis model to compare 


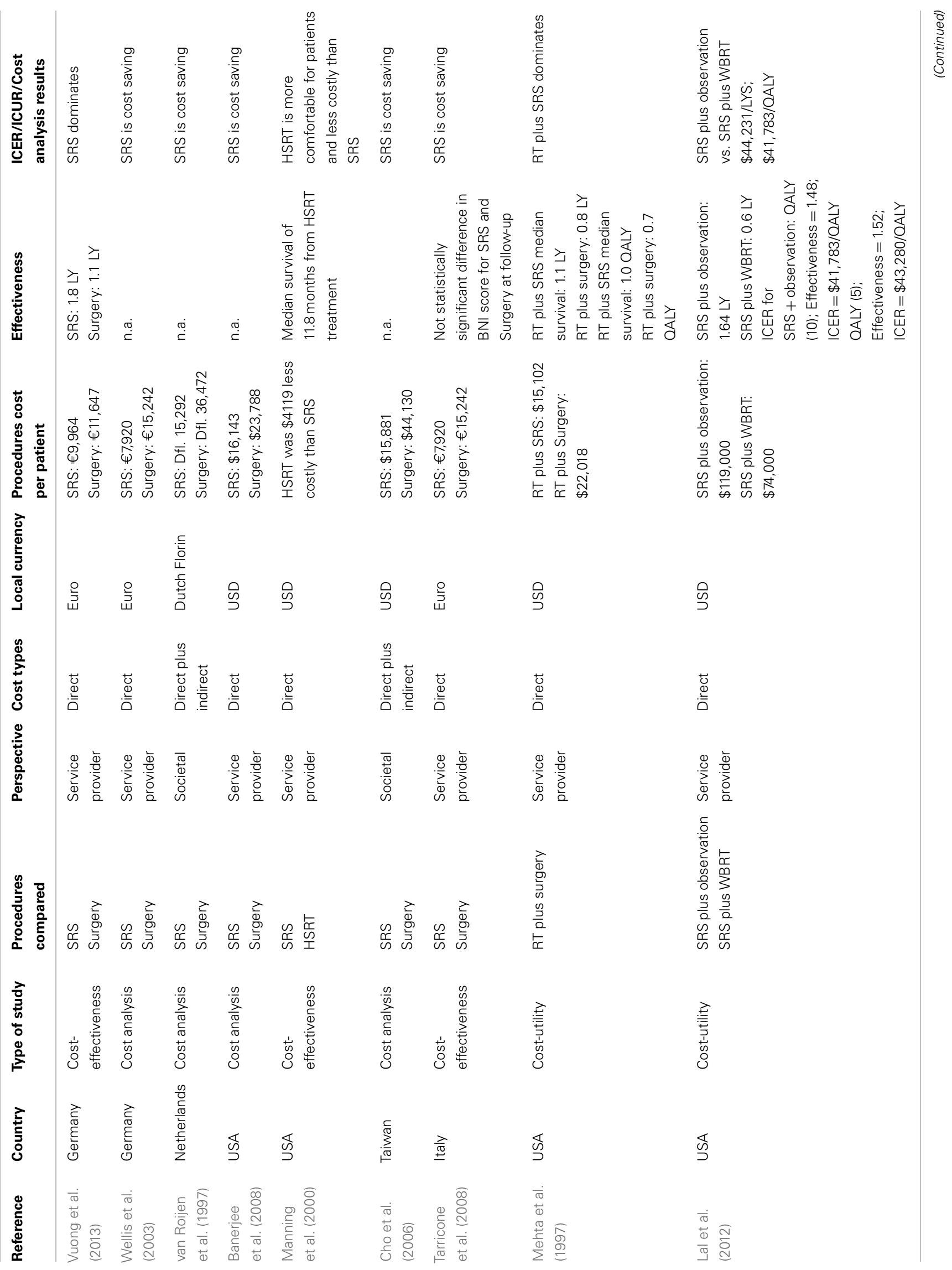




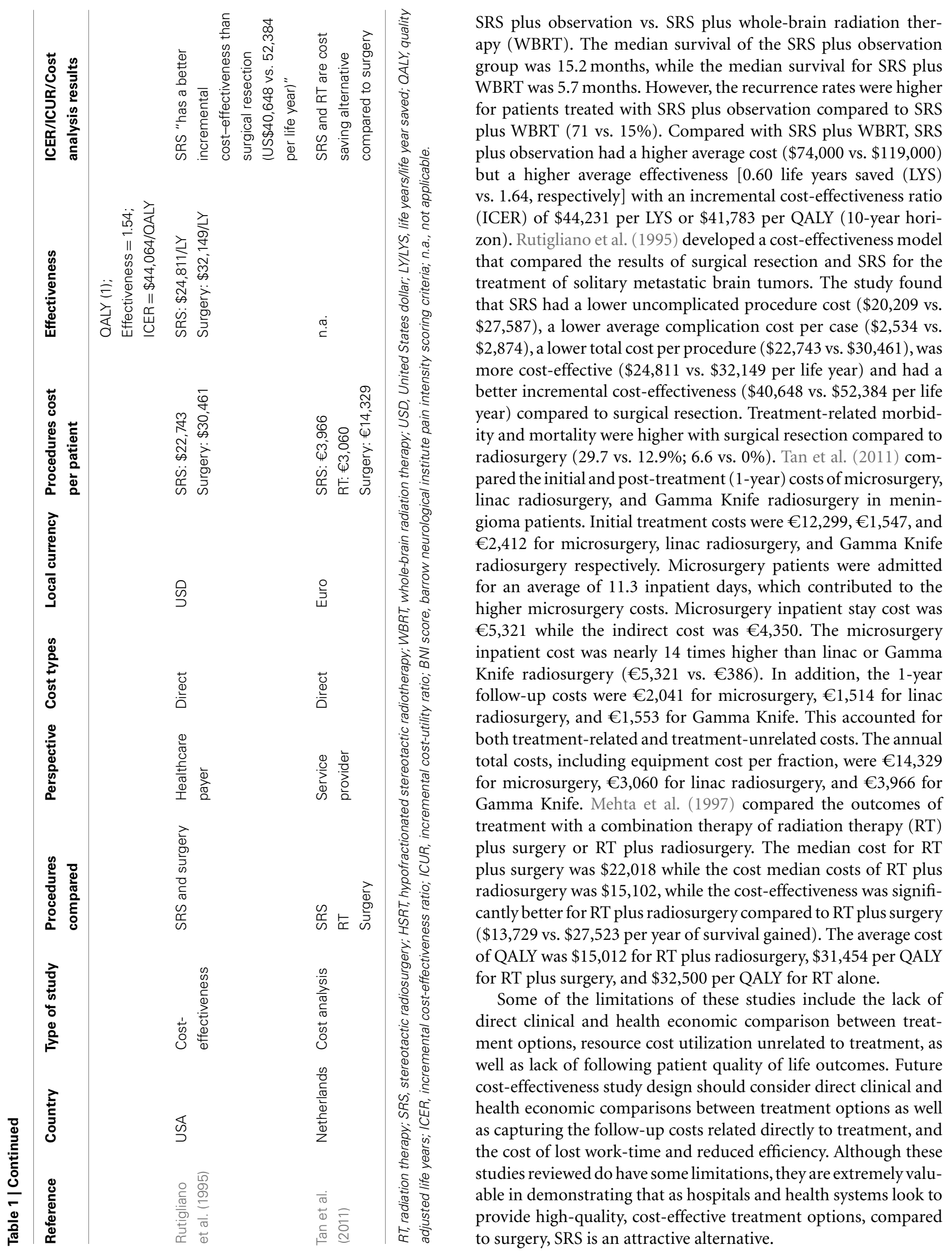




\section{SPINE}

Although spine radiosurgery is a well-developed extracranial application of SRS and SBRT, and considerable efficacy and safety data have been published, there is limited data on the costeffectiveness of the procedure (Table 2). In comparing external beam radiation therapy (EBRT) to SBRT for spinal metastases, Haley et al. (2011) found that the total cost to treat 100 patients with SBRT (including a $9 \%$ retreatment rate) was $\$ 842,420$, while the cost to treat $30 \mathrm{~Gy}$ in 10 fractions (including a $23 \%$ retreatment rate) was $\$ 676,309$ and the cost to treat 20 Gy in 5 fractions (including a $23 \%$ retreatment rate) was $\$ 499,911$. As noted, although SBRT was more costly than EBRT, patients treated with EBRT had higher levels of acute toxicities and were more likely to require additional interventions at the treated sites. Papatheofanis et al. (2009) constructed a Markov model to simulate outcomes of patients undergoing non-chemotherapeutic interventions - either CyberKnife SRS or EBRT - for metastatic spinal tumors. Patients treated with CyberKnife SRS gained an additional net health benefit of 0.08 QALY while the CyberKnife SRS cost was $\$ 11,812$ and EBRT was $\$ 13,745$, a difference of $\$ 1,933$.

The main limitations of these studies were the lack of headto-head comparative clinical and health economic data across therapy options and the fact that side effect treatments varied across patients.

Future trials should capture clinical and health economic data as well as quality of life indicators across all treatment options. The studies reviewed clearly demonstrate that SRS and SBRT provide clinicians with an additional cost-effective treatment option for spinal metastases that has better short-term results and comparable long-term results to EBRT.

\section{LUNG}

While surgical resection is the standard of care for many patients with non-small cell lung cancer (NSCLC), the location of the tumor and age and health status of patients with lung cancer often dictate whether they can undergo surgery. For those patients who are not surgical candidates, conventional RT and, more recently, SBRT, are treatment options. For many elderly patients with comorbid conditions such as emphysema and COPD, breath holding or controlled breathing (which may be required for RT delivered without tumor motion management capabilities) further reduces their options (Table 3). Lanni et al. (2011) compared the clinical and cost outcomes of SBRT, 3-dimensional conformal RT (3DCRT), and intensity modulated radiation therapy (IMRT) for the treatment of medically inoperable NSCLC. The treatment cost, calculated using the charge cost from the institution for the technical and professional components, for 35 fractions of 3DCRT was $\$ 55,705, \$ 136,570$ for 35 fractions of IMRT, and $\$ 52,471$ for 4 fractions of SBRT. The actual cost for a 35-fraction 3DCRT ranged from $\$ 50,000$ to $\$ 61,000$, while the actual cost of a 4 -fraction SBRT ranged from $\$ 41,000$ to $\$ 57,000$. At a median potential follow-up of up to 36 months, SBRT had higher overall survival compared to 3DCRT (71 vs. $42 \%$ ). Sher et al. (2011) developed a Markov model comparing SBRT, 3DCRT, and radiofrequency ablation (RFA) for 65-year-old men with medically inoperable NSCLC. In the base-case analysis, RFA, 3DCRT, and SBRT had a mean cost per QALY of $\$ 44,648 / 1.45, \$ 48,842 / 1.53$, and $\$ 51,133 / 1.91$, 
respectively. The ICER for SBRT over 3DCRT was $\$ 6,000 /$ QALY and $\$ 14,100 /$ QALY for SBRT over RFA. Compared to RFA and 3DCRT, SBRT had lower 3-year local recurrence, regional recurrence, and distant metastases rates. Puri et al. (2012) compared the cost-effectiveness of surgical intervention and SBRT in high-risk patients with stage I NSCLC. The median survival with surgery was 4.1 years, and the 4 -year survival was $51.4 \%$. With SBRT, the median survival was 2.9 years, and the 4 -year survival was $30.1 \%$. The cause-specific survival was identical between the two groups, and the difference in overall survival was not statistically significant. Nevertheless, SBRT was estimated to have a mean expected survival of 2.94 years at a cost of $\$ 14,153$ and mean expected survival with surgery was 3.39 years at a cost of $\$ 17,629$, for an ICER of $\$ 7,753$.

Limitations across these studies included the fact that the cost analysis was modeled from a Payer's perspective, rather than a societal or combined perspective. In addition, since these studies were retrospective, survival benefits may not have been fully captured across all therapy options. Ongoing cost-effectiveness studies should be done prospectively and not only capture the clinical outcomes of the different treatment options, but also quality of life measures. Given the positive clinical and health economic outcomes, SBRT provides a cost-effective and clinically effective outpatient and non-invasive therapy option for patients with NSCLC compared to conventional RT and RFA, while surgery remains the first treatment option in terms of cost-effectiveness.

\section{PROSTATE}

There are many different treatment options available to men diagnosed with localized prostate cancer including a variety of radiation therapies - 3DCRT, IMRT, proton therapy, SBRT, brachytherapy (HDR and LDR) - as well as surgical options - open, laparoscopic, and robotic (Table 4). Using a Markov model, Parthan et al. (2012) compared the cost-effectiveness of SBRT, IMRT, and proton therapy. The work-time lost due to treatment for SBRT, IMRT, and proton therapy was 10,90 , and $100 \mathrm{~h}$, respectively. From a payer perspective, SBRT dominated both IMRT and proton therapy (SBRT: cost $\$ 24,873$; QALY 8.11; IMRT: cost $\$ 33,068$; QALY 8.05; proton therapy: cost $\$ 69,094$; QALY 8.06). From a societal perspective, SBRT dominated both IMRT and proton therapy (SBRT: cost \$25,097; QALY 8.11; IMRT: cost \$35,088; QALY 8.05; proton therapy: cost \$71,339; QALY 8.06). Hodges et al. (2012) also utilized a Markov model to compare the cost-effectiveness of SBRT and IMRT. The model assumed IMRT costs of $\$ 29,530$ and SBRT costs of $\$ 14,315$. Results showed that the mean cost and QALYs for SBRT and IMRT were \$22,152 and 7.9 years and $\$ 35,431$ and 7.9 years, respectively.

Some of the limitations of these two studies include the limited long-term SBRT data for localized prostate cancer, thus potentially causing the current study models to inaccurately estimate SBRT clinical values. Future studies should focus not only on acute and late toxicity and long-term (5+ year) biochemical diseasefree survival, but also focus on including cost and quality of life measures.

Collectively, these studies demonstrated that SBRT is a cost saving treatment option for localized prostate cancer.

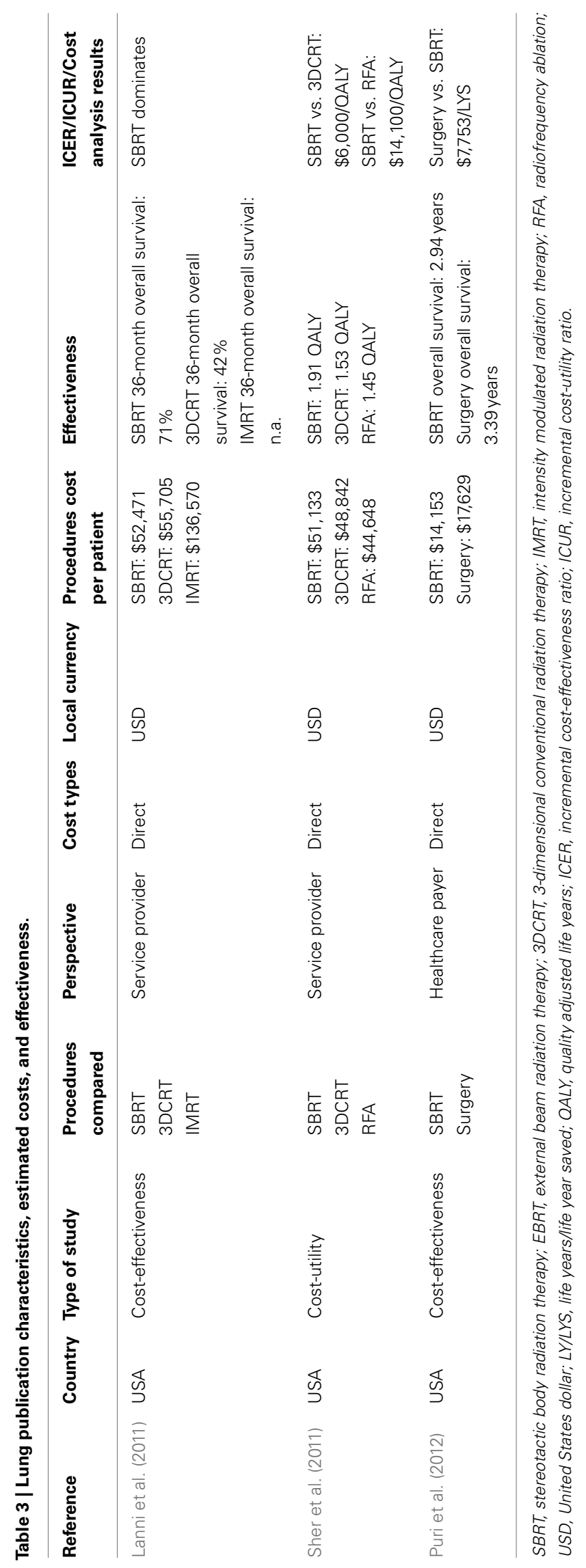




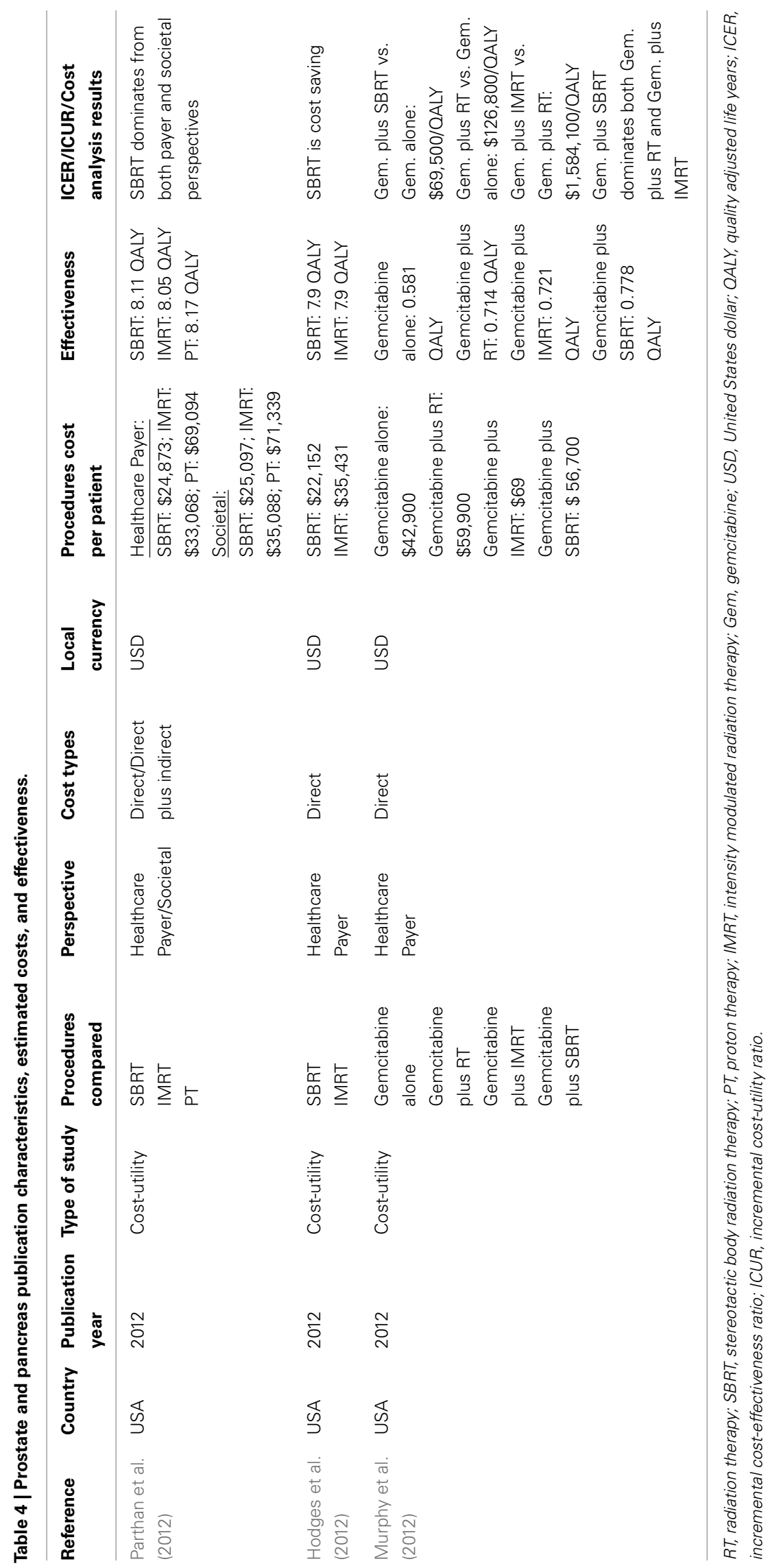




\section{PANCREAS}

Recent studies, including the Eastern Cooperative Oncology Group study E4201, demonstrated improved survival when chemotherapy is combined with RT for patients with pancreatic cancer (Table 4). Murphy et al. (2012) compared the costeffectiveness of four different therapies - gemcitabine, gemcitabine plus conventional RT, gemcitabine plus IMRT, and gemcitabine plus SBRT. The base-case cost of gemcitabine alone, gemcitabine plus SBRT, gemcitabine plus RT, and gemcitabine plus IMRT was $\$ 42,900, \$ 56,700, \$ 59,900$, and $\$ 69,500$, respectively. Overall, SBRT increased life expectancy by 0.20 QALY at an increased cost of $\$ 13,700$ compared with gemcitabine alone (ICER $=\$ 69,500$ per QALY). In the base-case analysis, gemcitabine plus SBRT dominated the more costly and less effective options of gemcitabine plus RT and gemcitabine plus IMRT. The study concluded that IMRT exceeds what society considers cost-effective in the treatment of locally advanced pancreatic cancer.

A limitation of this study was that the Markov model was used to compare preliminary results from phase 3 clinical trials (gemcitabine and gemcitabine plus RT in E4201) with phase 2 clinical data (gemcitabine plus SBRT). In addition, the model assumed actual costs and quality of life outcomes about supportive care for patients with pancreatic cancer. Future research needs should continue to capture the clinical outcomes but also add quality of life and cost measures. This will allow researchers to combine the clinical and health economic results in future publications.

\section{REFERENCES}

Adler, J. R. Jr., Gibbs, I. C., Puataweepong, P., and Chang, S. D. (2006). Visual field preservation after multisession cyberknife radiosurgery for perioptic lesions. Neurosurgery 59, 244-254; discussion 244-254.

Banerjee, R., Moriarty, J. P., Foote, R. L., and Pollock, B. E. (2008). Comparison of the surgical and followup costs associated with microsurgical resection and stereotactic radiosurgery for vestibular schwannoma. J. Neurosurg. 108, 1220-1224.

Blomgren, H., Lax, I., Naslund, I., and Svanstrom, R. (1995). Stereotactic high dose fraction radiation therapy of extracranial tumors using an accelerator. Clinical experience of the first thirty-one patients. Acta Oncol. 34, 861-870.

Chang, S. D., Gibbs, I. C., Sakamoto, G. T., Lee, E., Oyelese, A., and Adler, J. R. Jr. (2005). Staged stereotactic irradiation for acoustic neuroma. Neurosurgery 56, 1254-1261; discussion 1261-1253.

Cho, D. Y., Tsao, M., Lee, W. Y., and Chang, C. S. (2006). Socioeconomic costs of open surgery and gamma knife radiosurgery for benign cranial base tumors. Neurosurgery 58, 866-873; discussion 866-873.
Haley, M. L., Gerszten, P. C., Heron, D. E., Chang, Y. F., Atteberry, D. S., and Burton, S. A. (2011). Efficacy and cost-effectiveness analysis of external beam and stereotactic body radiation therapy in the treatment of spine metastases: a matched-pair analysis. J. Neurosurg. Spine 14, 537-542.

Hamilton, A. J., Lulu, B. A., Fosmire, H., Stea, B., and Cassady, J. R. (1995). Preliminary clinical experience with linear acceleratorbased spinal stereotactic radiosurgery. Neurosurgery 36, 311-319.

Hodges, J. C., Lotan, Y., Boike, T. P., Benton, R., Barrier, A., and Timmerman, R. D. (2012). Costeffectiveness analysis of stereotactic body radiation therapy versus intensity-modulated radiation therapy: an emerging initial radiation treatment option for organconfined prostate cancer. J. Oncol. Pract. 8, e31s-e37s.

Lal, L. S., Byfield, S. D., Chang, E. L., Franzini, L., Miller, L., Arbuckle, R., et al. (2012). Cost-effectiveness analysis of a randomized study comparing radiosurgery with radiosurgery and whole brain radiation metastases. Am. J. Clin. Oncol. 35, 45-50. therapy in patients with 1 to 3 brain

Based on the data reviewed, chemotherapy plus SBRT increased life expectancy compared to gemcitabine alone at a cost potentially acceptable by today's standards.

\section{CONCLUSION}

In our review of the current state of the research, SRS and SBRT have clearly demonstrated their clinical value and economic sustainability, often in comparison to long-standing and well-accepted treatment options. From a patient perspective, SRS and SBRT provide a patient-friendly treatment option compared to other treatment options such as conventional RT, especially those who live in a rural setting or a great distance from treatment centers. SRS and SBRT also offer a treatment option that is non-invasive and can be completed in the outpatient setting, thus potentially freeing up valuable inpatient hospital resources as well as allowing patients to resume their normal daily activity as quickly as possible. Both from payer and societal perspectives, the clinical and cost-effectiveness of SRS and SBRT have been demonstrated to reduce health system utilization (medication, retreatment, etc.) and minimize indirect costs, thus saving payers additional financial resources, and reducing the strain on the workforce. In times of increasing resource constraint cost-effective and cost saving techniques could be crucial for healthcare systems in order to maintain their sustainability in the long run. Identifying such techniques will require continued coupling of robust clinical research with economic data.

Lanni, T. B. Jr., Grills, I. S., Kestin, L. L., and Robertson, J. M. (2011). Stereotactic radiotherapy reduces treatment cost while improving overall survival and local control over standard fractionated radiation therapy for medically inoperable non-small-cell lung cancer. Am. J. Clin. Oncol. 34, 494-498.

Lee, W. Y., Cho, D. Y., Lee, H. C., Chuang, H. C., Chen, C. C. Liu, J. L., et al. (2009). Outcomes and cost-effectiveness of gamma knife radiosurgery and whole brain radiotherapy for multiple metastatic brain tumors. J. Clin. Neurosci. 16, 630-634.

Lindquist, C. (1989). Gamma knife surgery for recurrent solitary metastasis of a cerebral hypernephroma: case report. Neurosurgery 25 802-804.

Manning, M. A., Cardinale, R. M., Benedict, S. H., Kavanagh, B. D., Zwicker, R. D., Amir, C., et al. (2000). Hypofractionated stereotactic radiotherapy as an alternative to radiosurgery for the treatment of patients with brain metastases. Int. J. Radiat. Oncol. Biol. Phys. 47, 603-608.

Mehta, M., Noyes, W., Craig, B., Lamond, J., Auchter, R., French, M., et al. (1997). A cost-effectiveness and cost-utility analysis of radiosurgery vs. resection for single-brain metastases. Int. J. Radiat. Oncol. Biol. Phys. 39, 445-454.

Murphy, J. D., Chang, D. T., Abelson, J., Daly, M. E., Yeung, H. N., Nelson, L. M., et al. (2012). Cost-effectiveness of modern radiotherapy techniques in locally advanced pancreatic cancer. Cancer 118, 1119-1129.

Papatheofanis, F. J., Williams, E., and Chang, S. D. (2009). Cost-utility analysis of the cyberknife system for metastatic spinal tumors. Neurosurgery 64, A73-A83.

Parthan, A., Pruttivarasin, N., Davies, D., Taylor, D. C., Pawar, V., Bijlani, A., et al. (2012). Comparative cost-effectiveness of stereotactic body radiation therapy versus intensity-modulated and proton radiation therapy for localized prostate cancer. Front. Oncol. 2:81. doi:10.3389/fonc.2012.00081

Puri, V., Crabtree, T. D., Kymes, S., Gregory, M., Bell, J., Bradley, J. D., et al. (2012). A comparison of surgical intervention and stereotactic body radiation therapy for stage I lung cancer in highrisk patients: a decision analysis. J. Thorac. Cardiovasc. Surg. 143, 428-436. 
Rutigliano, M. J., Lunsford, L. D., Kondziolka, D., Strauss, M. J., Khanna, V., and Green, M. (1995). The cost effectiveness of stereotactic radiosurgery versus surgical resection in the treatment of solitary metastatic brain tumors. $\mathrm{Neu}$ rosurgery 37, 445-453; discussion 453-445.

Sher, D. J., Wee, J. O., and Punglia, R. S. (2011). Cost-effectiveness analysis of stereotactic body radiotherapy and radiofrequency ablation for medically inoperable, early-stage nonsmall cell lung cancer. Int. J. Radiat. Oncol. Biol. Phys. 81, e767-e774.

Tan, S. S., Van Putten, E., Nijdam, W. M., Hanssens, P., Beute, G. N., Nowak, P. J., et al. (2011). A microcosting study of microsurgery, LINAC radiosurgery, and gamma knife radiosurgery in meningioma patients. J. Neurooncol. 101, 237-245.

Tarricone, R., Aguzzi, G., Musi, F., Fariselli, L., and Casasco, A. (2008). Cost-effectiveness analysis for trigeminal neuralgia: cyberknife vs. microvascular decompression. Neuropsychiatr. Dis. Treat. 4, 647-652.

van Roijen, L., Nijs, H. G., Avezaat, C. J., Karlsson, G., Linquist, C., Pauw, K. H., et al. (1997). Costs and effects of microsurgery versus radiosurgery in treating acoustic neuroma. Acta Neurochir. (Wien) 139, 942-948.

Vuong, D. A., Rades, D., Van Eck, A. T., Horstmann, G. A., and Busse, R. (2013). Comparing the cost-effectiveness of two brain metastasis treatment modalities from a Payer's perspective: stereotactic radiosurgery versus surgical resection. Clin. Neurol. Neurosurg. 115, 276-284.

Wellis, G., Nagel, R., Vollmar, C., and Steiger, H. J. (2003). Direct costs of microsurgical management of radiosurgically amenable intracranial pathology in Germany: an analysis of meningiomas, acoustic neuromas, metastases and arteriovenous malformations of less than $3 \mathrm{~cm}$ in diameter. Acta Neurochir. (Wien) 145, 249-255.

Conflict of Interest Statement: The authors declare that the research was conducted in the absence of any commercial or financial relationships that could be construed as a potential conflict of interest.
Received: 07 January 2013; accepted: 27 March 2013; published online: 08 April 2013.

Citation: Bijlani A, Aguzzi G, Schaal DW and Romanelli P (2013) Stereotactic radiosurgery and stereotactic body radiation therapy cost-effectiveness results. Front. Oncol. 3:77. doi 10.3389/fonc.2013.00077

This article was submitted to Frontiers in Radiation Oncology, a specialty of Frontiers in Oncology.

Copyright (C) 2013 Bijlani, Aguzzi, Schaal and Romanelli. This is an open-access article distributed under the terms of the Creative Commons Attribution License, which permits use, distribution and reproduction in other forums, provided the original authors and source are credited and subject to any copyright notices concerning any third-party graphics etc. 